\title{
Effect of Hybrid Data Compression Technique on the Diagnostic Accuracy of Region of Interest (ROI) on MRI Image of a Spine Disc Prolapse
}

\author{
Richard O. Oyeleke \\ Department of Computer \\ science, \\ University of Lagos, \\ Akoka, Nigeria.
}

\author{
Adetunji P. Adewole \\ Department of Computer \\ science, \\ University of Lagos, \\ Akoka, Nigeria.
}

\author{
Florence A. Oladeji \\ Department of Computer \\ science, \\ University of Lagos, \\ Akoka, Nigeria
}

\begin{abstract}
The cost of transmitting and archiving medical images are quite prohibitive due to their large sizes especially in digital radiology system such as: picture archiving and communication systems and teleradiology. In order to reduce storage requirements and improve transmission rate, there is need for compression. Usually, radiologists are only interested in the abnormal region of the image (known as the region of interest) in making diagnosis and interpretations; hence, this work investigates the effect of hybrid data compression technique on the diagnostic accuracy of region of interest (ROI) on magnetic resonance image (MRI) of a spine disc prolapsed. We extract the ROI from the original image and apply lossless Wavelet-Based Compression (WBC) on the ROI while the remainder image known as the non-region of interest is compressed using discrete cosine transform (DCT). A compression ratio of 7:1 was achieved. Finally, the diagnostic accuracy of the compressed ROI image was evaluated subjectively by a group of 30 evaluators comprising of 20 radiologists and 10 Radiographers. The results obtained show a $100 \%$ acceptance of the compressed ROI for healthy diagnosis and interpretation.
\end{abstract}

\section{General Terms}

Data compression technique

\section{Keywords}

Image compression, medical image, region of interest, image evaluation, diagnostic accuracy

\section{INTRODUCTION}

The demand for transmission bandwidth and storage space in the digital radiology environment continues to outstrip the capabilities of available technologies. Hence, the need for efficient data compression techniques for cost-effective medical image transmission (Sumathy \& Pallavi, 2010). Compression of digital medical images can greatly improve their transmission, reduce storage requirements and processing. The huge sizes of digital imaging data, have continued to encourage the adoption of teleradiology and picture archiving and communication systems (PACS) in radiology departments (Cosman, Gray, \& Olsen, 2010). Generally, data compression schemes are categorised into two: "lossless" and "lossy" compression techniques. In lossless compression, image quality is preserved as that of the original image although with distinguishable size usually with compression ratio of $2: 1$. In contrast, lossy compression gives an approximation of the original image data thereby resulting to loss of image quality but a better compression ratio is obtained as compared to lossless compression schemes (Rehna \& Jeya, 2011). Medical professionals have opted for lossless compression of digital medical images since the compressed images offer same quality as the original image. Reason not farfetched as any form of degradation in quality of clinically important regions of the image known as region of interest (ROI) may lead to ill-diagnosis. This paper aim to evaluate and investigate the effect of hybrid data compression technique on the diagnostic accuracy of region of interest on magnetic resonance image of a spine disc prolapse.

\section{METHODOLOGY}

\subsection{Hybrid Data Compression Technique}

First, we classify the image into regions known as the ROI and non-region of interest (NROI) based on their clinical relevance for diagnosis. After the classification of the image regions, the ROI is separated from the spine disc prolapse image. This was achieved by calling imfreehand function into our ROI extractive function written using MATLAB version 7.14 (R2012a). Since classifying ROI may be relative, freehand selection allows a radiologist to easily define the ROI. It is also important to note that the accuracy of the ROI as defined by the radiologist will affect efficiency of this technique.

In the work of (Soundarya \& Bhavani, 2012), they compare two hybrid coding techniques using Integer Wavelet Transform (IWT) for compression of ROI while Discrete Cosine Transform (DCT) and fractal compression were used for compressing non-region of interests respectively. Similarly, (Kumar, Sharma, \& Ayub, 2012) employ a hybrid compression technique using Feed Forward Neural Network (FFN) for ROI and Set Partioning in Hierachical Trees (SPIHT) on the NROI image. Basically, our work employs a hybrid of a lossless Wavelet-Based Compression (WBC) for ROI and Discrete Cosine Transform (DCT) on the NROI.

\subsection{Region of Interest (ROI) Compression}

Once the ROI is extracted, it is then compressed using lossless Wavelet-Based Compression (WBC). WBC have been used in the field of medical imaging since it does not degrade the image quality, in other words, there is no loss of data when image is compressed (Lee, 2007). The WBC working 
principle is based on the image decomposition. In other words, the original image is decomposed hierarchically into approximate and detail images (coefficients) which contain the information needed to be reconstructed back to the next higher resolution level. The approximation is the low frequency component while the detail images are the high frequency components. However, the low frequency component is regarded to be more important than the high frequency component. The approximate and detail images are obtained by applying a low pass filter and high pass filter for each row of data in the image (Satish, 2001). We used a decomposition level of 1 so that majority of the image energy is compacted into the approximate image, as a higher decomposition level allow more energy to be lost which compromises the image quality. Further, thresholding is done. This modifies the coefficients to produce more zeros, since for some of the decomposed reference images, many of the wavelet coefficients are approximately or equal to zero. This in turn yields a large number of consecutive zero's which optimizes storage space and ultimately allow for a higher rate of compression (Lees, 2002).

\subsection{Non-Region of Interest (NROI) Compression}

The NROI is compressed using DCT. It is a compression technique which conforms to the JPEG standard. Its primary aim is to reduce the required number of bits to represent an image (Radha \& Shruthi, 2013). It is preferred in the field of medical imaging for compression as it allows for the degree of data loss to be controlled. In other words, radiologist can specify the level at which the image should be degraded especially for the non-region of interest (NROI) which might be clinically less relevant for making diagnosis. DCT compression is achieved into phases: The first being the quantization stage, where a lossy compression is performed. The transformed image is represented with a lesser number of bits by discarding some image data known as the higher frequency components which are considered to be less important than the low frequency components. The quality of the compressed image is determined in this phase since user can specify value for the compression level desired. A higher value gives a better image quality with low compression ratio and a lower value gives a poorer image quality with a higher compression ratio. The second compression stage is called the entropy coding stage. This involves a lossless compression of the quantized coefficients. Its working principle is based on the designation of fewer bits to represent colours that occur frequently in an image and more bits to those that occur infrequently. It is based on Huffman coding technique (Gupta \& Garg, 2010).

\section{EVALUATION OF COMPRESSED MEDICAL IMAGE}

One important challenge with the compression of medical image is the evaluation of its quality. Generally, two approaches exist viz: objective and subjective measures. The objective measure can be done by computing the Mean Square Error (MSE), which measures punctual variations of the image intensity by averaging the squared differences between couples of corresponding pixels. The efficiency of the objective measure on image quality depends strongly on its accordance with subjective measure. More so, a lower MSE does not always translate to better quality in the compressed image since it is not a subjective measure of the image quality (Saffor et al., 2010). In this work, subjective measure of evaluation was used. The viewers focus on the difference between reconstructed image and the original image, they notice such details where information loss cannot be accepted (Ghrare et al., 2008). This test emphasizes the examination of fidelity and at the same time considers image intelligibility. We employed a subjective measure for evaluation of the Compressed ROI image. This measure is based on a rating scale given (i.e. mean opinion score). 30 copies of a self-designed questionnaire containing an image rating scale were distributed among 30 evaluators comprising radiologists and radiographers who were chosen randomly at the Radiodiagnosis Department, Lagos University Teaching Hospital, Idi-Araba, Lagos, Nigeria.

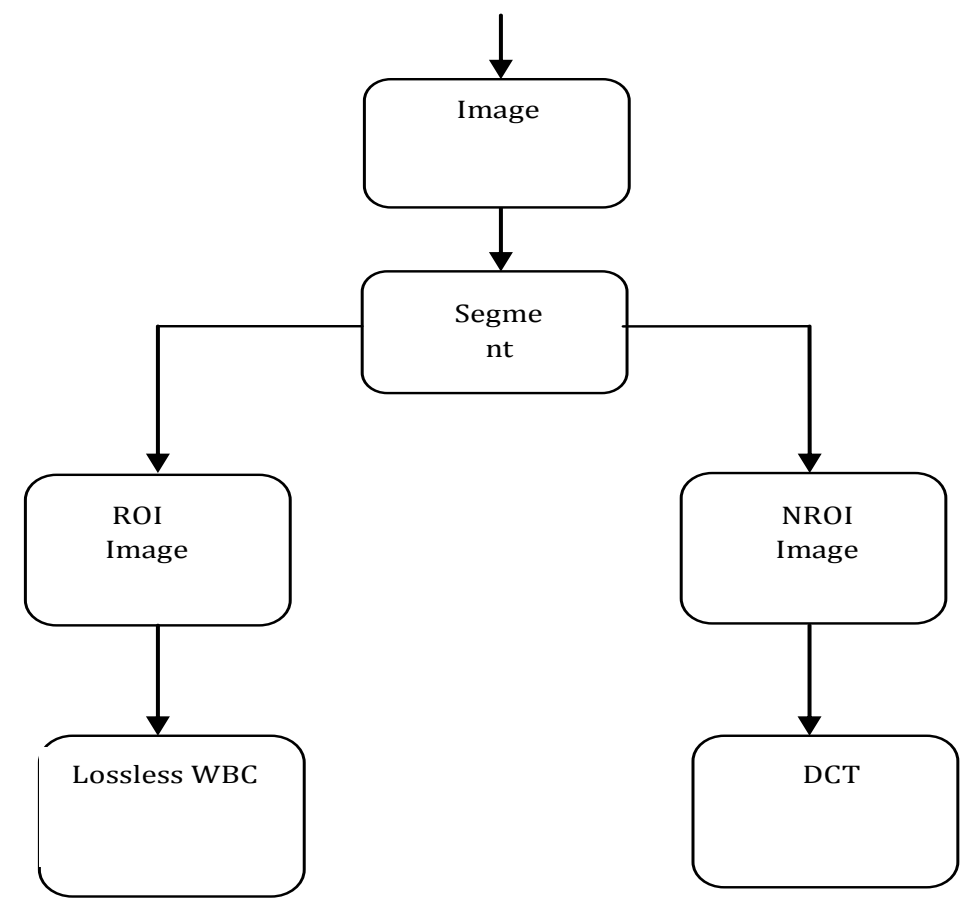

Fig 1: Hybrid data compression technique flowchart 
International Journal of Applied Information Systems (IJAIS) - ISSN : 2249-0868

Foundation of Computer Science FCS, New York, USA

Volume 7-No.5, July 2014 - www.ijais.org

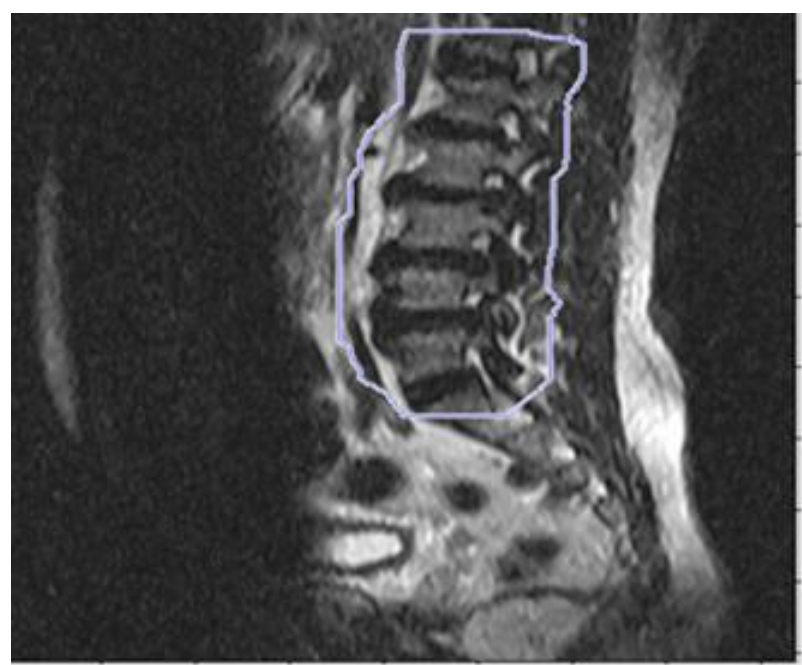

Fig. 2. Region of interest highlighted for segmentation

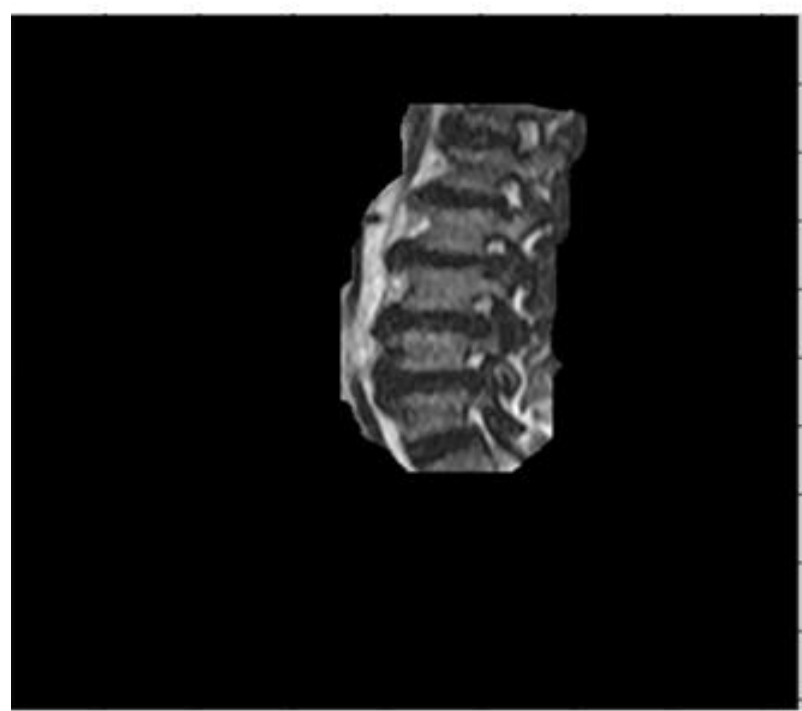

Fig 3. Extracted region of interest (ROI) image

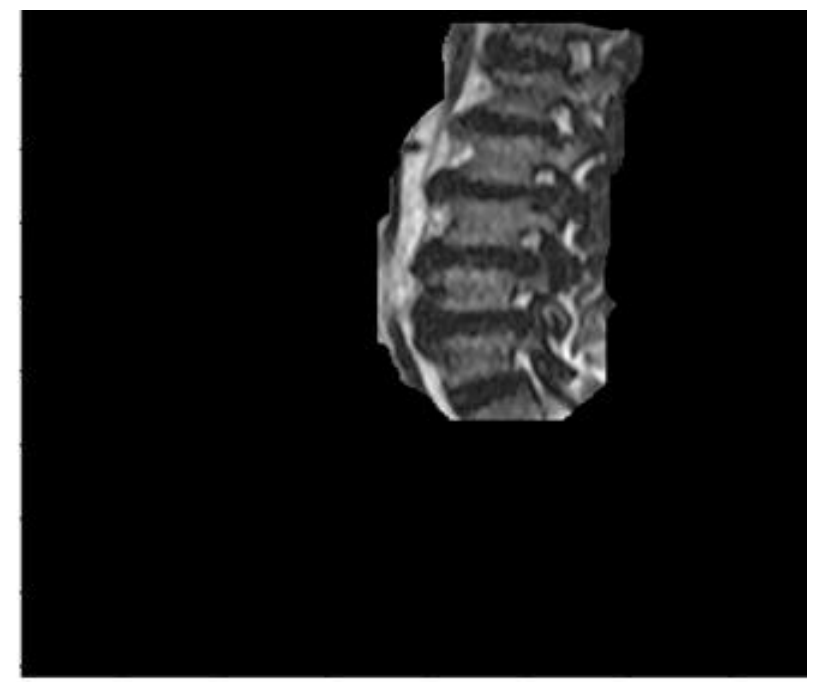

Fig. 4. Compressed Region of interest (ROI) image

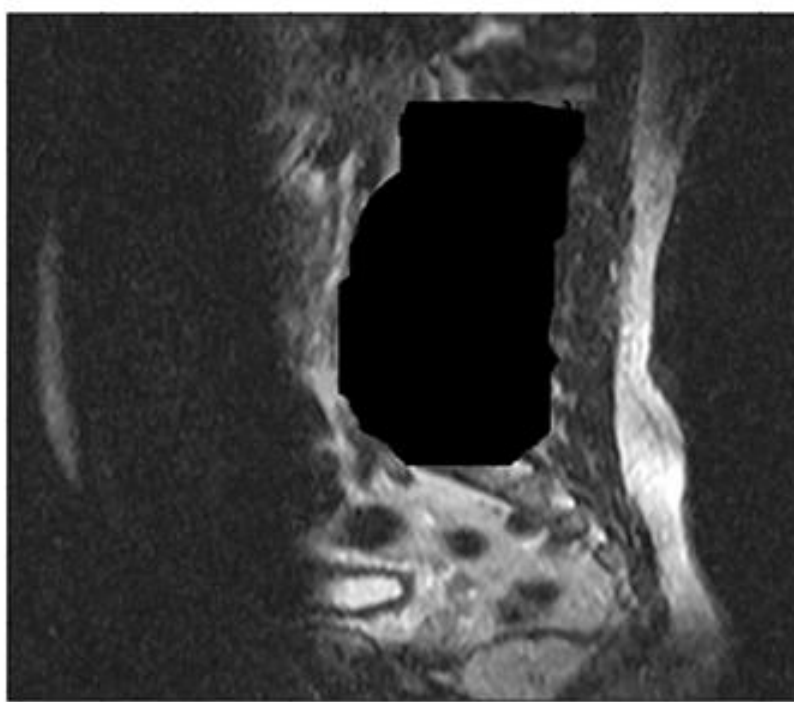

Fig. 5. Non-region of interest (NRON image

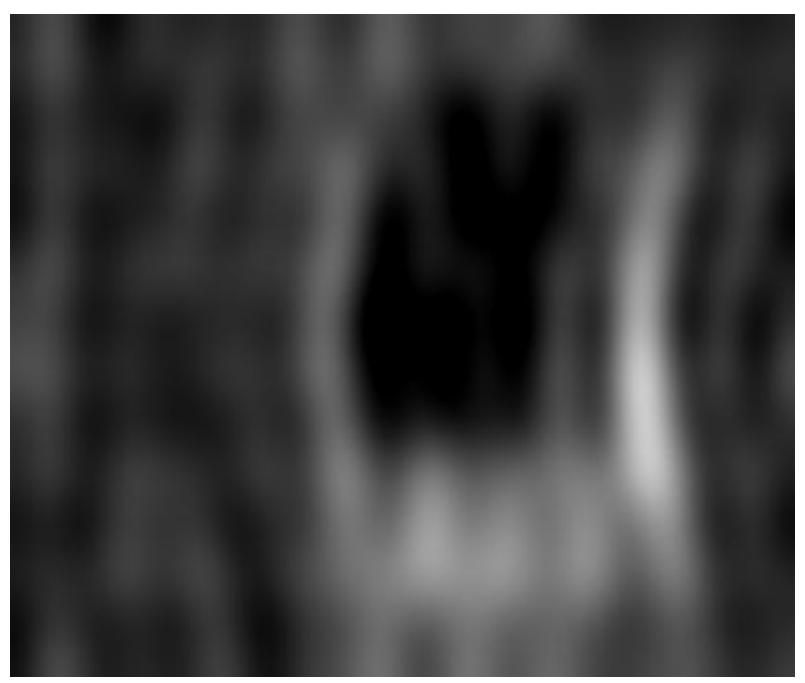

Fig. 5: Compressed NROI image using DCT at compression coefficient of 50 


\section{RESULTS AND DISCUSSION}

Table 1, represents the image rating scale calibrated into two parts based on the diagnostic implication of the subjective ratings made by each evaluator. The first part of the calibration spans from scale 1-2 which is classified as "Not Acceptable for healthy diagnosis and interpretations". This implies that any subjective rating made by each evaluator which falls on this class is not acceptable and should be rejected. The second part of the calibration spans from scale 3-5, classified as "Acceptable for healthy diagnosis and interpretations", hence, implies that ratings which fall on this classification are reliable and efficient for diagnosis, and hence, should be accepted.

Table 2, represents the subjective rating of the ROI image made by 30 evaluators From this table, it was shown that out of the thirty evaluators: eight (8) of the evaluators rated the compressed ROI as "Highly diagnostic" which represents $26.7 \%$ of the evaluators. This implies that $26.7 \%$ of the evaluators can make diagnosis and interpretations with high level of accuracy as they would with the original image of the spine disc prolapse. More so, fifteen (15) of the evaluators rated the compressed ROI as "Diagnostic", which represents $50.0 \%$ of the evaluators. This therefore implies that $50.0 \%$ of the evaluators can make accurate diagnosis and interpretations from the compressed ROI. Further, seven (7) of the evaluators rated the compressed ROI as "Moderately diagnostic" which represents $23.3 \%$ of the evaluators. This implies that this group of evaluators can still make diagnosis and interpretations with the based on the compressed ROI, although moderate. In addition, none of the evaluators rated the compressed ROI as either "Poorly diagnostic" or "Not diagnostic". Finally, the hybrid data compression technique gives a compression ratio of 7:1, and since ratings made by the evaluators all fall on the first part of the calibration of the image rating scale as shown in Table 1, these sum up to a $100 \%$, hence, it therefore implies the image possesses healthy trait which suffice for accurate and reliable diagnosis and interpretations, hence, should be accepted.

Table 1. subjective image rating scale for compressed roi image

\begin{tabular}{lll}
\hline \hline Score & $\begin{array}{c}\text { Degree of } \\
\text { accuracy for } \\
\text { diagnosis }\end{array}$ & \multicolumn{1}{c}{ Acceptability } \\
\hline 5 & Highly Diagnostic & $\begin{array}{l}\text { Acceptable for healthy } \\
\text { diagnosis and interpretations } \\
\text { Acceptable for healthy } \\
\text { diagnosis and interpretations }\end{array}$ \\
3 & Diagnostic & $\begin{array}{l}\text { Acceptable for healthy } \\
\text { diagnosis and interpretations }\end{array}$ \\
2 & Doderately & $\begin{array}{l}\text { Not acceptable for healthy } \\
\text { diagnosis and interpretations }\end{array}$ \\
1 & Not Diagnostic & $\begin{array}{l}\text { Not acceptable for healthy } \\
\text { diagnosis and interpretations }\end{array}$
\end{tabular}

Table 2. results of subjective rating of compressed roi image

\begin{tabular}{lcc}
\hline \multicolumn{1}{c}{$\begin{array}{c}\text { Degree of } \\
\text { accuracy }\end{array}$} & Frequency & Percentage \% \\
\hline $\begin{array}{l}\text { Highly } \\
\text { Diagnostic }\end{array}$ & 8 & 26.7 \\
$\begin{array}{l}\text { Diagnostic } \\
\text { Moderately } \\
\text { Diagnostic }\end{array}$ & 15 & 50.0 \\
$\begin{array}{l}\text { Poorly } \\
\text { Diagnostic }\end{array}$ & 0 & 23.3 \\
$\begin{array}{l}\text { Not } \\
\text { Diagnostic }\end{array}$ & 0 & 0 \\
\end{tabular}

Total

\section{CONCLUSION}

In this work, we investigated the effect of hybrid data compression technique on the diagnostic accuracy of the region of interest on an MRI image of a spine disc prolapsed. The image was segmented into two parts, namely: ROI and NROI. The ROI was compressed using lossless wavelet-based compression while the NROI was compressed using DCT. The diagnostic accuracy of the compressed ROI image was evaluated subjectively by a group of 30 evaluators comprising of 20 radiologists and 10 Radiographers. The results obtained show a $100 \%$ acceptance of the compressed ROI for healthy diagnosis and interpretation.

Finally, a compression ratio of 7:1 was achieved which implies that the hybrid data compression technique will aid faster transmission of medical images and save network bandwidth and aid long term archiving of medical images in digital radiology systems especially in picture archiving and communication systems (PACS) and teleradiology applications for optimized storage.

\section{REFERENCES}

[1] P.C. Cosman, R.M. Gray and R.A. Olsen, "Evaluating quality of compressed medical images: SNR, subjective rating, and diagnostic accuracy", Retrieved from: https://www.zotero.org/rudedude/items/itemKey/SS2T2 HU4, 2010.

[2] M. Gupta and A.K. Garg, "Analysis Of Image Compression Algorithm Using DCT", International Journal of Engineering Research and Applications (IJERA), vol. 2, issue 1, pp.515-521, 2012.

[3] S.E. Ghrare, M.A.M. Ali, M. Ismail, and K. Jumari, "The Effect of Image Data Compression on the Clinical Information Quality of Compressed Computed Tomography Images for Teleradiology Applications", European Journal of Scientific Research, vol.23, No.1, pp.6-12. 2008.

[4] V. Kumar, J.Sharma and S. Ayub, "Image Compression using FFN for ROI and SPIHT for background, International Journal of Computer Applications", Volume 46- No.18, 2012. 
[5] T.H. Lee, "Introduction to Medical Image Compression Using Wavelet Transform" , Graduate Institute of Communication Engineering, National Taiwan University, 2007.

[6] K. Lees, "Image Compression Using Wavelets",2002.

[7] K.A. Radha, and G. Shruthi, "JPEG Encoder using Discrete Cosine Transform \& Inverse Discrete Cosine Transform", IOSR Journal of Electronics and Communication Engineering (IOSR-JECE) .,vol. 5, issue 4, pp. 51-56, 2013.

[8] V.J. Rehna, and K.M.K. Jeya, "Hybrid Approaches to Image Coding: A Review": (IJACSA), International Journal of Advanced Computer Science and Applications, vol. 2, no. 7., 2011.

[9] A. Saffor, A.B. Ramil, K. Ng, D. Dowsett, "Objective and Subjective Evaluation of Compressed Computed
Tomography (CT) Images", Retrieved Available from: http: isup.com/IJRA/2/2/6159, 2010.

[10] K. S. Satish, "Classifying image data", Retrieved from:http://www.debugmode.com/imagecmp/classify.ht $\mathrm{m}, 2001$.

[11] Y.S. Sumathy and A. Pallavi, "Region of Interest (ROI) based Medical Image Compression and reliable transmission with application to CT images (Liver images)", Visvesvaraya Technological University, Belgaum-590018, R. N. Shetty Institute of Technology, Bangalore, 2009.

[12] G. Soundarya,, and S. Bhavani, "Comparison of Hybrid Codes for MRI Brain Image Compression", Research Journal of Applied Sciences, Engineering and Technology, vol4, pp. 24, 2012. 